\title{
Spectroscopic ellipsometry of metal phthalocyanine thin films
}

\author{
Aleksandra B. Djurišić, Chung Yin Kwong, Tsz Wai Lau, Zheng Tong Liu, \\ Hoi Sing Kwok, Lillian Sze Man Lam, and Wai Kin Chan
}

\begin{abstract}
Optical functions of cobalt phthalocyanine, nickel phthalocyanine (NiPc), and iron phthalocyanine $(\mathrm{FePc})$ have been determined by use of spectroscopic ellipsometry in the spectral range 1.55-4.1 eV (300-800 $\mathrm{nm})$. The samples were prepared by evaporation onto glass and silicon substrates. The optical functions were determined by point-to-point fit. Absorption spectra were also measured. The index-ofrefraction data for $\mathrm{NiPc}$ and $\mathrm{FePc}$ are reported for the first time to our knowledge. Good agreement with the experimental spectra was obtained for all three materials. (c) 2003 Optical Society of America OCIS codes: $310.6860,160.4890,120.2130$.
\end{abstract}

\section{Introduction}

Phthalocyanines are porphyrin derivatives that are characterized by high degrees of symmetry, planarity, and electron delocalization. ${ }^{1}$ Phthalocyanines (Pcs) have attracted much attention for applications in organic optoelectronic devices such as organic solar cells, ${ }^{1-3}$ in organic field effect transistors, ${ }^{4}$ in organic light-emitting diodes in which Pcs can be used as a hole transport layers ${ }^{5,6}$ or emitting layers ${ }^{7}$; and in gas sensors. ${ }^{8,9}$ For modeling and design of optoelectronic devices, the optical function of each layer should be known. Optical properties of solids are usually described in terms of the complex dielectric function $\varepsilon(\omega)=\varepsilon_{1}(\omega)+i \varepsilon_{2}(\omega)$ or the complex index of refraction $N(\omega)=n(\omega)+i k(\omega)$. Copper phthalocyanine $(\mathrm{CuPc})$ has been the most widely studied metal Pc. Dielectric functions and indices of refraction of $\mathrm{CuPc}$ were studied experimentally by use of reflectance and transmission measurements, ${ }^{10}$ absorption coefficient measurement with Kramers-Kronig analysis, ${ }^{11}$ and spectroscopic ellipsometry. ${ }^{12,13}$ Unlike

A. B. Djurišić (dalek@eee.hku.hk), C. Y. Kwong, T. W. Lau, L. S. M. Lam, and W. K. Chan are with the University of Hong Kong, Pokfulam Road, Hong Kong, China. A. B. Djurišić and C. Y. Kwong are with the Department of Electrical Engineering and L. S. M. Lam and W. K. Chan are with the Department of Chemistry; A. B. Djurišić is also with the Department of Physics. Z. T. Liu and H. S. Kwok are with the Department of Electrical Engineering, Hong Kong University of Science and Technology, Clearwater Bay, Hong Kong, China.

Received 22 May 2003; revised manuscript received 8 July 2003. 0003-6935/03/316382-06\$15.00/0

(C) 2003 Optical Society of America
CuPc, other metal Pcs have not been extensively studied. Investigation of cobalt phthalocyanine $(\mathrm{CoPc})$ by spectroscopic ellipsometry has been reported. ${ }^{14}$ Absorption spectra of various Pc films, ${ }^{15-17}$ as well as vapor phases and solutions in different solvents, ${ }^{18,19}$ have also been reported.

The growth and morphology of the various Pc films have been extensively studied. ${ }^{20-35}$ Pc films can exist in several crystalline polymorphs. Most common polymorphs are metastable $\alpha$ and stable $\beta$ phases. The main differences between polymorphs lie in the tilt angle of the molecules within the columns (stacks of molecules with molecular planes parallel to one another) and the mutual arrangement of the columns. Transformation from phase $\alpha$ to phase $\beta$ by exposure to solvent vapors ${ }^{25,29,30}$ or by annealing or deposition at higher temperature ${ }^{26-28,31,32}$ has been reported. The absorption spectra of different polymorphs of the same Pc compound differ significantly..$^{15,27,28}$ The polymorphism of Pcs significantly influences the preferred orientation in Pc layers. The substrate also seems to play a role in the orientation of the deposited films. ${ }^{21-24,26}$ It has been demonstrated that different Pcs show evident isomorphism with one another ${ }^{23}$ and that deposition on mica at temperatures above $400 \mathrm{~K}$ results in oriented films. ${ }^{23,24}$ Wachtel et al. ${ }^{24}$ found that the stability of the films may be substrate dependent and that lithium Pc films deposited on glass substrates at room temperature were stable over several months, whereas those on $\mathrm{NaCl}$ substrates showed marked changes when they were exposed to ambient conditions. For low-temperature substrates, quasiepitaxial growth of thin fluorine bridged aluminum 
Pc polymer by molecular-beam epitaxy was obtained on alkali halide substrates, whereas epitaxial growth did not occur on silicon and quartz. ${ }^{21}$ Oriented films with two different $b$-axis (stacking axis) orientations, depending on the substrate's temperature, have been grown on copper substrates. ${ }^{12,33}$ For Si substrates maintained at room temperature, the preferred orientation of evaporated CuPc films less than $100 \mathrm{~nm}$ thick is in the [200] direction, with the $\alpha$ form dominant. ${ }^{26}$ With increasing thickness, randomization of the orientation of crystallites occurs; the $\alpha$ form is still prevalent. ${ }^{26}$ For CuPc films deposited on glass substrates, the preferential orientation of the crystallites appeared to depend on the deposition rate and on whether the substrate was rotating ([110] orientation) or nonrotating ([100] orientation). ${ }^{22}$ Bayliss et $a l .{ }^{32}$ reported that the $\alpha$ form of the metal-free Pc $\mathrm{H}_{2} \mathrm{Pc}$ deposited on microscope slides at room temperature consists of small spherical particles that are randomly oriented, whereas oriented domains in the films have been found only for the $\beta$ form obtained by annealing the $\alpha$ form. The $\beta$ form obtained by direct evaporation onto substrates heated to $330{ }^{\circ} \mathrm{C}$ showed elongated crystallites similar to those in the $\beta$ form obtained by annealing, but randomly oriented. ${ }^{32}$ Highly oriented CuPc film fabricated by molecularbeam deposition onto quartz glass substrates at low deposition rates has been demonstrated. ${ }^{34}$

We report spectroscopic ellipsometry measurements of $\mathrm{CoPc}$, nickel phthalocyanine $(\mathrm{NiPc})$, and iron phthalocyanine $(\mathrm{FePc})$ thin films deposited on glass and silicon substrates at room temperature. Because the deposition is performed at room temperature, we expected that the films would be polycrystalline and predominantly of the $\alpha$ form. The spectroscopic ellipsometry measurements were performed in the spectral range 1.55-4.1 eV (300-800 $\mathrm{nm})$. Samples were modeled as isotropic layers, as was reported to be adequate for $\mathrm{CuPc}$ samples less than 80-100 nm thick. ${ }^{36}$ Although each Pc molecule has an intrinsic anisotropy that is due to its planar structure, for random orientation of crystallites in a polycrystalline film an isotropic model is sufficient. Atomic-force microscopy (AFM) and absorption measurements were also performed. The samples for absorption measurements were deposited onto quartz substrates.

The paper is organized as follows: in section 2, experimental details are described, and Section 3 presents results and discussions. Finally, conclusions are presented in Section 4.

\section{Experimental Details}

Pc powders were purchased from Strem Chemicals. First, silicon substrates were cleaned in deionized (DI) water by a standard four-cycle dump-rinse procedure. After that, the wafer was cleaned for $10 \mathrm{~min}$ in an $\mathrm{H}_{2} \mathrm{SO}_{4}: \mathrm{H}_{2} \mathrm{O}_{2}$ bath at $120^{\circ} \mathrm{C}$. Another fourcycle dump-rinse in DI water was performed, followed by $\mathrm{HF}$ cleaning ( $\mathrm{HF}: \mathrm{H}_{2} \mathrm{O}$ 1:50) for $30 \mathrm{~s}$ to remove native oxide. After rinsing in DI water and drying, the wafers were immediately loaded into an evaporator to minimize formation of another oxide layer. Microscope slide glass and quartz substrates were cleaned in an ultrasonic bath for $30 \mathrm{~min}$, rinsed with DI water, and dried in flowing nitrogen. Before cleaning, the back side of the glass substrate was roughened to suppress back side reflections for spectroscopic ellipsometry (SE) measurement. For absorption and AFM measurements, the films deposited on quartz substrates were used. The films were evaporated in high vacuum. The sample holder can hold up as many as four substrates, so the films fabricated on microscope slides, silicon, and quartz were deposited in the same deposition process. Pressure during evaporation was of the order $10^{-4}$ $\mathrm{Pa}$. The evaporation rate was $0.1-0.2 \mathrm{~nm} / \mathrm{s}$. The distance from source to film was $\sim 23 \mathrm{~cm}$ to ensure uniformity of film thickness, and the substrate holder could be rotated. The thickness of the films was controlled by a quartz thickness monitor.

The film thickness was measured by a step profiler. Pc films are absorbing in the spectral region of interest. Absorption is weak in the 450-550-nm spectral region, but assuming a Cauchy model for the refractive index and disregarding the coefficient of extinction in this spectral region can cause errors in the thickness determined from fitting of SE data. The film thickness was also verified by AFM. A multimode scanning-probe microscope from Digital Instruments was used for AFM measurements. The thickness was measured at 10 points along the step to minimize the influence of possible errors caused by variation in thickness around the step. In addition to revealing film thickness, AFM also yielded a surface-roughness estimate. The $\mathrm{rms}$ surface roughness in all films was below $3.5 \mathrm{~nm}$; the mean roughness was below $2 \mathrm{~nm}$. The film thickness was $48 \mathrm{~nm}$ for CoPc, $56 \mathrm{~nm}$ for $\mathrm{FePc}$, and $45 \mathrm{~nm}$ for NiPc.

Absorption measurements were performed on a Perkin-Elmer Lambda $20 \mathrm{UV} / \mathrm{Vis}$ spectrophotometer. The SE data in the 300-800 nm spectral range were measured with a Jobin Yvon UVISEL phasemodulated ellipsometer. Phase-modulated ellipsometers are comparable to the more frequently used rotating analyzer ellipsometers in terms of attainable precision. ${ }^{37}$ The incidence angle used was $70^{\circ}$. The samples exhibited good uniformity, which we verified by performing measurements at different areas of the sample on both glass and silicon substrates. Also, the samples did not exhibit any in-plane anisotropy, as we verified by rotating the sample $90^{\circ}$ and repeating the measurement. Point-by-point fitting of the measured spectra was performed. We used a simulated annealing algorithm to fit the data. ${ }^{38}$ The fitting was performed simultaneously for glass and silicon substrates. The objective function used was

$$
\begin{aligned}
F_{i}= & \left\lfloor\Phi[\tan (\Psi)]_{\text {glass }}+\Phi[\tan (\Psi)]_{\mathrm{Si}}\right. \\
& \left.+\Phi[\cos (\Delta)]_{\mathrm{glass}}+\Phi[\cos (\Delta)]_{\mathrm{Si}}\right\rfloor_{i}+\gamma_{i, i-1}, \\
\Phi(X)= & \left(X^{\exp }-X^{\text {calc }}\right)^{2}
\end{aligned}
$$


where $i$ denotes the $i$ th experimental data point, the subscripts glass and Si denote glass and silicon substrates, respectively, and the superscripts exp and calc denote experimental and calculated data. $\gamma$ is an optional penalty function that can be introduced to ensure the smoothness of the $n$ and $k$ obtained by limiting the allowed difference between values obtained in current $(i)$ and previous $(i-1)$ data points. We obtained this difference in the following manner: If the difference between $n$ and $k$ values in current and previous data points was larger than the specified allowed difference, $\gamma$ was set to a large positive constant.

Otherwise, $\gamma$ was set to zero. Because of the existence of multiple solutions, the use of such a penalty function to prevent unphysical abrupt changes in $n$ and $k$ is advisable. We determined the refractive indices of the substrates from the SE measurements of the substrates, instead of taking the data from the material database, to ensure improved accuracy of the determined $n$ and $k$ of the Pc films. For Si substrates, fitting with and without a native oxide layer was performed. There was no significant difference between the results obtained in these two cases. The result of simultaneous point-to-point fitting for both substrates shows somewhat inferior agreement with the experimental data compared with point-topoint fitting for one substrate only. However, reliability of the data obtained by simultaneous fitting for both substrates is considerably improved compared with data obtained by fitting for only one substrate because there are two unknowns and four conditions that need to be satisfied.

Correction for surface roughness was not made because individual points yield different thickness values for a rough layer. Fixing the thickness of the rough layer to the value averaged over a wavelength resulted in an inferior fit. We also attempted to model the optical functions of the Pc layer and then to fit the thickness of the Pc layer and the thickness of the rough layer. However, although numerous models of the optical functions of inorganic semiconductors are available, for organic materials the choice is typically limited to the Lorentz oscillator model and its modifications ${ }^{39,40}$ or the Forouhi-Bloomer model. ${ }^{41}$ It was shown previously that the conventional Lorentz model is inadequate for modeling optical functions of organic materials. ${ }^{39,40}$ The ForouhiBloomer model was used only in a very narrow spectral range (365-564 $\mathrm{nm}$ ) for a material that exhibited a single peak in the spectral range investigated. ${ }^{41}$ We attempted to use both the conventional Lorentz model and its modifications, with and without surface-roughness correction. In all cases, agreement with the experimentally determined $\tan (\Psi)$ and $\cos (\Delta)$ was inferior compared to that from point-topoint fitting. Therefore, here we present the results of optical functions obtained by point-to-point fitting without surface-roughness correction. We improved the reliability of the data by fitting the data for films on two substrates simultaneously. We also verified the quality of the result obtained by comparing the positions of the peaks in the calculated extinction coefficient spectrum with the measured absorption data.

\section{Results and Discussion}

Pc films grown upon room-temperature substrates typically consist of small spherical particles and are predominantly of $\alpha$ modification ${ }^{26,32}$ or possibly of the $x$ form. ${ }^{25}$ When the substrate temperature is increased, the size of the crystallites increases, some preferential ordering in neighboring domains begins, ${ }^{25}$ and finally at higher temperatures the $\beta$ form is obtained. ${ }^{32}$ Deposition at temperatures below room temperature results in significantly smoother films. ${ }^{25,35}$ Vincett et $a l .{ }^{35}$ believed that the films were in the $\alpha$ form, though at temperatures below $-50{ }^{\circ} \mathrm{C}$ the films may have been partially amorphous. Brinkmann et al. ${ }^{25}$ however, stated that films grown at temperatures of $5{ }^{\circ} \mathrm{C}-25{ }^{\circ} \mathrm{C}$ have disordered, i.e., close to amorphous, structures based on diffuse diffraction rings. Pan et al. ${ }^{42}$ have found that vanadyl Pc films deposited at room temperature and exposed to flowing nitrogen for $50 \mathrm{~h}$ are partially amorphous. In a previous analysis of the optical functions of $\mathrm{CuPc}$ by variable-angle spectroscopic ellipsometry, Debe ${ }^{12}$ investigated $\mathrm{Pc}$ films grown at $0{ }^{\circ} \mathrm{C}$ and $70{ }^{\circ} \mathrm{C}$, which he assumed were well oriented, and deduced the approximate optical functions that correspond to parallel and perpendicular orientation with respect to stacking axis $b$. The possibility that the film deposited at low temperature may not have been well uniaxially oriented has been acknowledged, ${ }^{12}$ but the possibility of phase change or of amorphousness of the films grown at low temperature has not been considered. In the research reported here, all the films were grown upon room-temperature substrates, so it is reasonable to assume that the films consist of polycrystalline, predominantly $\alpha$ phase domains with little or no preferential orientation among the neighboring domains. Because the film thickness in all cases is less than $80-100 \mathrm{~nm}$, we have considered the films to be isotropic. ${ }^{36}$ For films $100-150 \mathrm{~nm}$ thick, an isotropic inner layer and an anisotropic outer layer should be assumed, whereas a film thicker than $150 \mathrm{~nm}$ can be treated as a single homogeneous anisotropic film. ${ }^{36}$

Figures 1-3 show $\tan (\Psi)$ and $\cos (\Delta)$ for $\mathrm{CoPc}, \mathrm{FePc}$, and NiPc films, respectively, deposited onto glass and silicon substrates. Figure 4 shows the absorption spectra for $\mathrm{CoPc}, \mathrm{NiPc}$, and $\mathrm{FePc}$. In the spectral region investigated, two distinct absorption bands can be observed, a band near $2 \mathrm{eV}(620 \mathrm{~nm} ; Q$ band) and one near $3.5 \mathrm{eV}$ (350 nm; $B$ or Soret band). Both bands have been assigned to $\pi \rightarrow \pi^{*}$ transitions of the macrocyclic $\pi$ system consisting of $\mathrm{C}$ and $\mathrm{N}$ atoms. ${ }^{11,13,18}$ The excitons that contribute to the absorption in these two bands are shown to be independent in $\mathrm{H}_{2}$ Pc. $^{7}$ There is little difference among the visible spectra of various metal Pcs. However, metal-dependent absorption bands can be observed near the infrared spectra of Pcs. ${ }^{18}$ Also, it has been suggested that both $Q$ and $B$ bands can be 

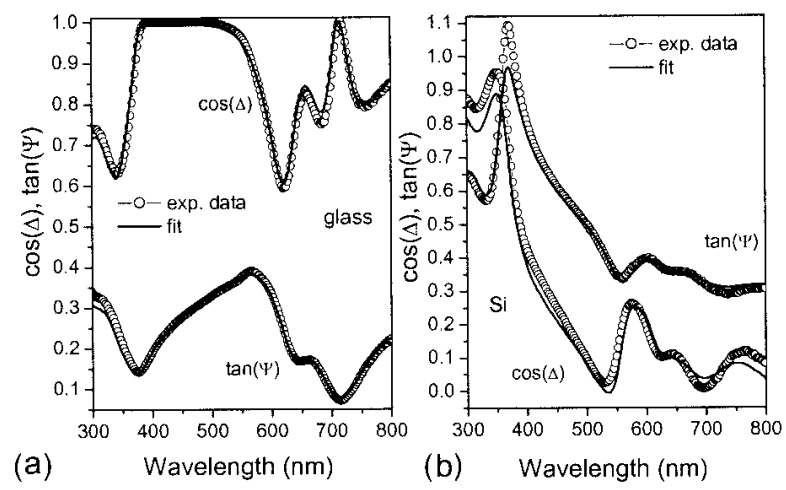

Fig. 1. $\operatorname{Tan}(\psi)$ and $\cos (\Delta)$ for $\operatorname{CoPc}$ films on glass and silicon substrates. The fit is a point-to-point fit; (a) glass substrate, (b) silicon substrate.

influenced by the metal-to-ligand charge-transfer bands in $\mathrm{CoPc}$ (Ref. 14), and the existence of a chargetransfer band at the red edge of the $Q$ band has been proposed. However, based on the small difference of the shift of absorption peak on the low-energy side of the $Q$ band of CuPc in solvents of different polarities (dimethyl sulfoxide and 1-chloronaphtalene), 18 it is reasonable to conclude that the charge transfer does not play a significant role in transitions on the lowenergy side of the $Q$ band. The exact identification
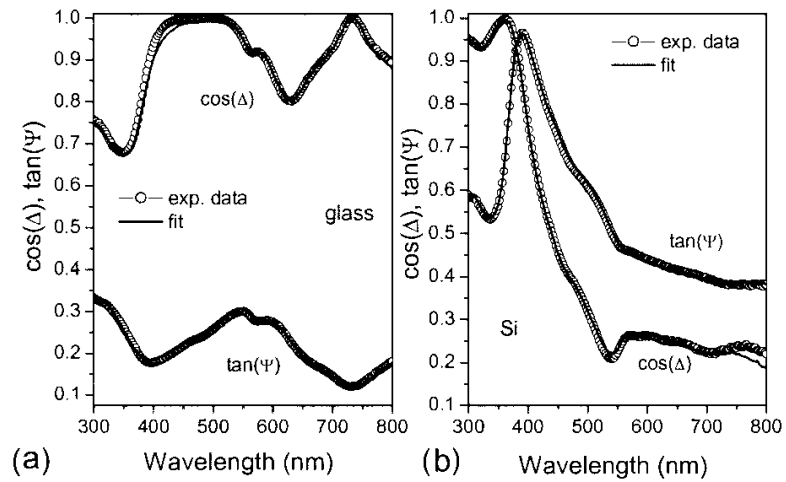

Fig. 2. $\operatorname{Tan}(\psi)$ and $\cos (\Delta)$ for FePc films on glass and silicon substrates. The fit is a point-to-point fit.
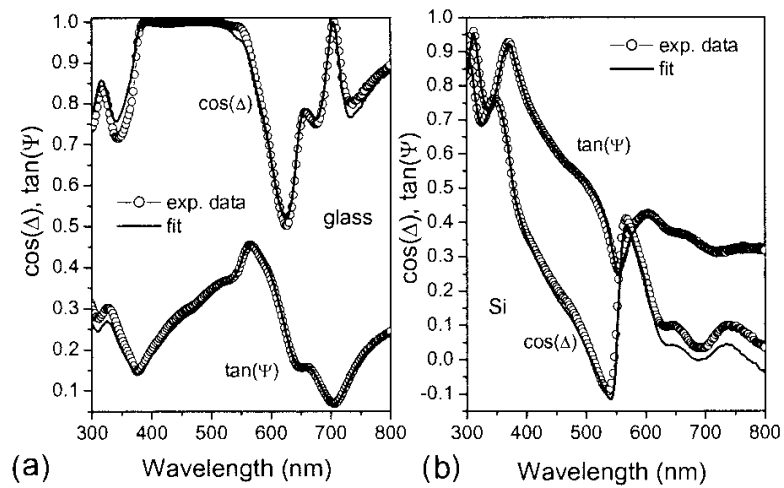

Fig. 3. $\operatorname{Tan}(\psi)$ and $\cos (\Delta)$ for NiPc films on glass and silicon substrates. The fit is a point-to-point fit: (a) glass substrate, (b) silicon substrate.

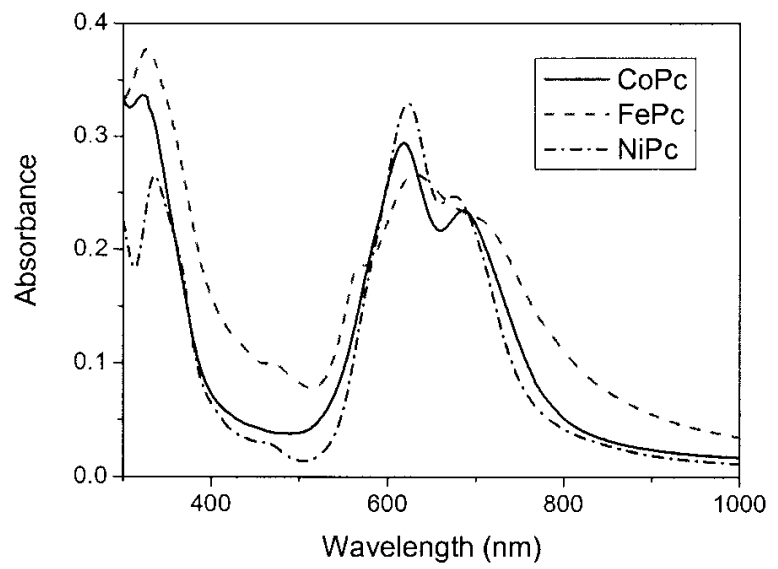

Fig. 4. Absorption spectra of $\mathrm{CoPc}, \mathrm{FePc}$, and NiPc.

of the origin of the observed peaks in the absorption spectrum of a $\mathrm{Pc}$ material is rather complex. $A b$ initio calculations of $\mathrm{Pc}$ molecules have been performed, and the calculated results are in good agreement with vapor phase spectra.43 However, the spectra of solutions and thin films in which interactions between individual molecules play a role are inherently more complicated.

Figure 5 shows the real and imaginary parts of the indices of refraction of $\mathrm{CoPc}, \mathrm{FePc}$, and NiPc. Good agreement between the peak positions in the calculated extinction coefficients and the measured absorption spectra can be observed. Chen et al. ${ }^{14}$ determined the index of refraction of CoPc films on silicon by spectroscopic ellipsometry in the spectral region from 550 to $800 \mathrm{~nm}$. Their extinction coefficient and absorption coefficient data show three peaks in this spectral region, and the value of the extinction coefficient is greater than 0.5 over the entire region, although it should be low at 550 and 800 $\mathrm{nm}$ according to absorption measurements reported in the literature that consistently have shown only two peaks. ${ }^{15,17}$ This difference may be due partly to the very thick film (greater than $600 \mathrm{~nm}$ ) investigated by Chen et al. ${ }^{14}$ It has been shown that for $\mathrm{CuPc}$ an anisotropic model is needed for analysis of

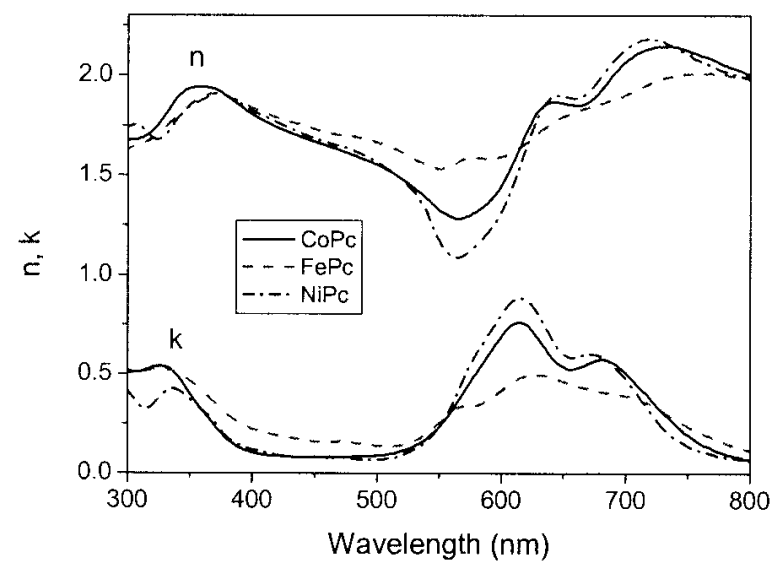

Fig. 5. Real (n) and imaginary (k) parts of the index of refraction of $\mathrm{CoPc}, \mathrm{FePc}$, and NiPc. 
the ellipsometry data of films thicker than $150 \mathrm{~nm}$. Lucia and Verderame ${ }^{15}$ identified two peaks in the $Q$ band of the absorption spectrum of CoPc, located at 1.82 and $2.01 \mathrm{eV}$ (680 and $616 \mathrm{~nm})$. Davidson ${ }^{17}$ identified peaks at 1.82 and $1.98 \mathrm{eV}(680$ and $626 \mathrm{~nm})$ in the $Q$ band and at $3.79 \mathrm{eV}(327 \mathrm{~nm})$ in the $B$ band. Our results are in good agreement with previously reported absorption spectra and with the absorption spectrum measured by us. We observed peaks at $1.82 \mathrm{eV}(680 \mathrm{~nm})$ and $2.02 \mathrm{eV}(615 \mathrm{~nm})$ in $k$ derived from $\mathrm{SE}$ and at $1.80 \mathrm{eV}(688 \mathrm{~nm})$ and $2.01 \mathrm{eV}(618$ $\mathrm{nm}$ ) in the absorption spectrum. In the $B$ band we observed peaks at $3.81 \mathrm{eV}(325 \mathrm{~nm})$ in $k$ and of 3.84 $\mathrm{eV}(323 \mathrm{~nm})$ in absorption.

For FePc, no previous data on the index of refraction were reported to our knowledge. Absorption spectra for FePc have been reported, ${ }^{16,17}$ and three peaks can be observed in the absorption spectra. Davidson ${ }^{17}$ obtained peaks at $1.73 \mathrm{eV}(717 \mathrm{~nm}), 1.98$ $\mathrm{eV}(626 \mathrm{~nm})$, and $2.19 \mathrm{eV}(566 \mathrm{~nm})$ in the $Q$ band and peaks at $3.51 \mathrm{eV}(353 \mathrm{~nm})$ and $3.83 \mathrm{eV}(324 \mathrm{~nm})$ in the $B$ band. Our results show maxima in the extinction coefficient at $1.74 \mathrm{eV}(713 \mathrm{~nm}), 1.98 \mathrm{eV}(625 \mathrm{~nm})$, and $2.18 \mathrm{eV}(570 \mathrm{~nm})$ in the $Q$ band and wide structure in the $B$ band with a maximum at $3.81 \mathrm{eV}(325 \mathrm{~nm})$, whereas in the absorption spectrum we observe peaks at $1.73 \mathrm{eV}(717 \mathrm{~nm}), 1.96 \mathrm{eV}(631 \mathrm{~nm}), 2.18 \mathrm{eV}$ $(568 \mathrm{~nm})$, and $3.79 \mathrm{eV}(327 \mathrm{~nm})$, is in good agreement with the absorption spectra reported in the literature.

Also for NiPc, we are not aware of any previous study of the complex index of refraction. Godlewski et al. ${ }^{44}$ obtained an absorption spectrum showing a two-peak structure and identified four transitions in the $Q$ band of NiPc, located at $1.76 \mathrm{eV}(704 \mathrm{~nm}), 1.83$ $\mathrm{eV}(678 \mathrm{~nm}), 1.98 \mathrm{eV}(626 \mathrm{~nm})$, and $2.02 \mathrm{eV}(614 \mathrm{~nm})$. Absorption measurements by Lucia and Verderame showed absorption maxima at $1.85 \mathrm{eV}(670 \mathrm{~nm})$ and $1.98 \mathrm{eV}(626 \mathrm{~nm})$, in good agreement with our results showing peaks at $1.84 \mathrm{eV}(673 \mathrm{~nm})$ and $2.02 \mathrm{eV}(615$ $\mathrm{nm})$ in the $Q$ band and $3.70 \mathrm{eV}(335 \mathrm{~nm})$ in the $B$ band. In the absorption spectrum, peaks at $1.84 \mathrm{eV}$ $(674 \mathrm{~nm}), 1.99 \mathrm{eV}(623 \mathrm{~nm})$, and $3.69 \mathrm{eV}(336 \mathrm{~nm})$ can be observed. However, the refractive-index minimum obtained near $2.19 \mathrm{eV}(566 \mathrm{~nm})$ is the lowest among the Pc films measured. There are several likely reasons for this observation. One could be surface roughness of the film. However, NiPc was the smoothest film obtained ( $\mathrm{rms}$ roughness, $2.4 \mathrm{~nm}$; mean roughness, $1.7 \mathrm{~nm}$ ), so it is not likely that surface roughness is causing the difference from other metal Pcs investigated. It has also been proposed that there are differences in refractive indices at film-air and film-glass interfaces in NiPc films. ${ }^{44}$ The second reason is possible anisotropy of the films. The refractive-index data determined by Schechtman and Spicer ${ }^{11}$ for a CuPc film with thickness of 23-45 $\mathrm{nm}$ grown at room temperature upon $\mathrm{LiF}$ substrates also show a dip in the refractive-index values to below 1.0 in the spectral region $2-2.5 \mathrm{eV}(495-620 \mathrm{~nm})$. This probably indicates a certain degree of preferential ordering in their samples, which is not surprising for alkali halide substrates. ${ }^{21}$ No in-plane anisotropy was detected when the sample was rotated and the ellipsometry measurement repeated, but this does not rule out the presence of anisotropy in the $z$-axis direction. Unfortunately, because the films are not likely to be ideally ordered, it is not possible to determine their anisotropy unambiguously. Further investigations are needed to resolve this issue.

\section{Conclusions}

Optical functions of $\mathrm{CoPc}, \mathrm{NiPc}$, and $\mathrm{FePc}$ thin films have been determined by use of spectroscopic ellipsometry in the spectral range 300-800 $\mathrm{nm}$. The films are likely to be polycrystalline with randomly oriented crystallites, so an isotropic model of the sample was adopted. The optical functions data were determined by simultaneous point-to-point fits of $\tan (\psi)$ and $\cos (\Delta)$ measured for films deposited upon glass and silicon substrates. Excellent agreement between experimental and calculated $\tan (\psi)$ and $\cos (\Delta)$ curves was obtained for all three materials. The agreement between the positions of the maxima in the imaginary part of the index of refraction and the absorption spectra reported in the literature and measured by us was good for all materials.

This study was supported by The Research Grants Council (RGC) of the Hong Kong Special Administrative Region, China (projects HKU 7096/00P and HKU 7075/01P), the University of Hong Kong seed funding research grant, and an RGC Co-operative Research Centers grant from the Hong Kong University of Science and Technology.

\section{References}

1. D. Wöhrle, L. Kreienhoop, and D. Schlettwein, "Phthalocyanines and related macrocycles in organic photovoltaic junctions," in Phthalocyanines: Properties and Applications, C. C. Leznoff and A. B. P. Lever, eds. (VCH, New York, 1996), pp. 219-284.

2. P. Peumans, V. Bulović, and S. R. Forrest, "Efficient photon harvesting at high optical intensities in ultrathin organic double-heterostructure photovoltaic diodes," Appl. Phys. Lett. 76, 2650-2652 (2000).

3. M. Pfeiffer, A. Beyer, B. Plönings, A. Nollau, T. Fritz, K. Leo, D. Schlettvein, S. Hiller, and D. Wöhrle, "Controlled $p$-doping of pigment layers by cosublimation: basic mechanisms and implications for their use in organic photovoltaic cells," Sol. Energy Mater. Sol. Cells 63, 83-99 (2000).

4. Z. Bao, A. J. Lovinger, and A. Dodabalapur, "Organic fieldeffect transistors with high mobility based on copper phthalocyanine," Appl. Phys. Lett. 69, 3066-3068 (1996).

5. J. Blochwitz, M. Pfeiffer, T. Fritz, and K. Leo, "Low voltage organic light emitting diodes featuring doped phthalocyanine as hole transport material," Appl. Phys. Lett. 73, 729-731 (1998).

6. X. Zhou, M. Pfeiffer, J. Blochwitz, A. Werner, A. Nollau, T. Fritz, and K. Leo, "Very-low-operating-voltage organic lightemitting diodes using a $p$-doped amorphous hole injection layer," Appl. Phys. Lett. 78, 410-412 (2001).

7. A. Fujii, Y. Ohmori, and K. Yoshino, "An organic infrared electroluminescent diode utilizing a phthalocyanine film," IEEE Trans. Electron Devices 44, 1204-1207 (1997).

8. T. Nagasawa, K. Murakami, and K. Watanabe, "Improvement in $\mathrm{NO}_{2}$-sensing characteristics of alpha-copper phthalocyanine 
thin films by the deposition on the hydrofluoric acid-treated glass substrates," Mol. Cryst. Liq. Cryst. 316, 389-392 (1998).

9. M. I. Newton, T. K. H. Starke, M. R. Willis, and G. McHale, " $\mathrm{NO}_{2}$ detection at room temperature with copper phthalocyanine thin film devices," Sens. Actuators B 67, 307-311 (2000).

10. T. Fritz, J. Hahn, and H. Böttcher, "Determination of the optical constants of evaporated dye layers," Thin Solid Films 170, 249-257 (1989).

11. B. H. Schechtman and W. E. Spicer, "Near infrared to vacuum ultraviolet absorption spectra and the optical constants of phthalocyanine and porphyrin films," J. Mol. Spectrosc. 33, 28-48 (1970).

12. M. K. Debe, "Variable angle spectroscopic ellipsometry studies of oriented phthalocyanine films. II. Copper phthalocyanine," J. Vac. Sci. Technol. A 10, 2816-2821 (1992).

13. A. Ritz and H. Lüth, "The electronic structure of GaP (110) and Cu-phthalocyanine overlayers studied by ellipsometry," Appl. Phys. A 31, 75-80 (1983).

14. Q. Chen, D. Gu, and F. Gan, "Ellipsometric spectra of cobalt phthalocyanine films," Physica B 212, 189-194 (1995).

15. E. A. Lucia and F. D. Verderame, "Spectra of polycrystalline phthalocyanines in the visible region," J. Chem. Phys. 48, 2674-2681 (1968).

16. H. Laurs and G. Heiland, "Electrical and optical properties of phthalocyanine films," Thin Solid Films 149, 129-142 (1987).

17. A. T. Davidson, "The effect of the metal atom on the absorption spectra of phthalocyanine films," J. Chem. Phys. 77, 168-172 (1982).

18. L. Edwards and M. Gouterman, "Porphyrins. XV. Vapor absorption spectra and stability: phthalocyanines," J. Mol. Spectrosc. 33, 292-310 (1970).

19. P. E. Fielding and A. G. MacKay, "Electrical conduction in the phthalocyanines. I. Optical properties," Aust. J. Chem. 17, $750-758$ (1964).

20. A. Schmidt, L. K. Chau, A. Back, and N. Armstrong, "Epitaxial phthalocyanine ultrathin films grown by organic molecular beam epitaxy (OMBE)," in Phthalocyanines: Properties and Applications, C. C. Leznoff and A. B. P. Lever, eds., (VCH, New York, 1996), pp. 307-341.

21. H. Hoshi, A. J. Dann, and Y. Maruyama, "The structure and properties of phthalocyanine films grown by the molecularbeam epitaxy technique. II. Ultraviolet/visible spectroscopic study," J. Appl. Phys. 67, 1845-1849 (1990).

22. B. Resel, M. Ottmar, M. Hanack, J. Keckes, and B. Leising, "Preferred orientation of copper phthalocyanine thin films evaporated on amorphous substrates," J. Mater. Res. 15, 934939 (2000).

23. N. Uyeda, M. Ashida, and E. Suito, "Orientation overgrowth of condensed polycyclic aromatic compounds vacuum-evaporated on cleaved face of mica," J. Appl. Phys. 36, 1453-1460 (1965).

24. H. Wachtel, J. C. Wittmann, B. Lotz, M. A. Petit, and J. J. Andre, "Anisotropic spin transport in oriented lithium phthalocyanine thin films," Thin Solid Films 250, 219-231 (1994).

25. M. Brinkmann, J. C. Wittmann, C. Chaumont, and J. J. Andre, "Effects of solvent on the morphology and crystalline structure of lithium phthalocyanine thin films and powders," Thin Solid Films 292, 192-203 (1997).

26. O. Berger, W. J. Fischer, B. Adolphi, S. Tierbach, V. Melev, and J. Schreiber, "Studies on phase transformations of $\mathrm{Cu}$ phthalocyanine thin films," J. Mater. Sci. Mater. Electron. 11, 331346 (2000).

27. J. H. Sharp and M. Lardon, "Spectroscopic characterization of a new polymorph of metal free phthalocyanine," J. Phys. Chem. 72, 3230-3235 (1968).

28. S. M. Bayliss, S. Heutz, G. Rumbles, and T. S. Jones, "Effect of annealing on the properties of thin films of free base phthalocyanine and perylene-3,4,9,10 tetracarboxylic dianhydride deposited by organic molecular beam deposition," Mater. Res. Soc. Symp. Proc. 560, 71-74 (1999).

29. F. Iwatsu, T. Kobayashi, and N. Uyeda, "Solvent effects on crystal growth and transformation of zinc phthalocyanine," J. Phys. Chem. 84, 3223-3230 (1980).

30. F. Iwatsu, "Size effects on the alpha-beta transformation of phthalocyanine crystals," J. Phys. Chem. 92, 1678-1681 (1988).

31. S. I. Shihub and R. D. Gould, "Studies of phase transformations in some metal phthalocyanine thin films using measurements of current as a function of temperature," Thin Solid Films 290-291, 390-394 (1996).

32. S. M. Bayliss, S. Heutz, R. Cloots, R. L. Middleton, G. Rumbles, and T. S. Jones, "Templating effects in the growth of metal-free phthalocyanine polymorphic double layers," Adv. Mater. 12, 202-206 (2000).

33. M. K. Debe, R. J. Poirier, and K. K. Kam, "Organic-thin-filminduced molecular epitaxy from the vapor phase," Thin Solid Films 197, 335-347 (1991).

34. M. Komiyama, Y. Sakakibara, and H. Hirai, "Preparation of highly ordered ultrathin films of copper(II) phthalocyanine on amorphous substrates by molecular beam deposition," Thin Solid Films 151, L109-L110 (1987).

35. P. S. Vincett, Z. D. Popovic, and D. McIntyre, "A novel structural singularity in vacuum-deposited thin films: the mechanism of critical optimization of thin film properties," Thin Solid Films 82, 357-376 (1981).

36. M. A. Barrett, Z. Borkowska, M. W. Humphreys, and R. Parsons, "Ellipsometry of thin films of copper phthalocyanine," Thin Solid Films 28, 289-302 (1975).

37. D. E. Aspnes, "Precision bounds to ellipsometer systems," Appl. Opt. 14, 1131-1136 (1975).

38. A. B. Djurišić, A. D. Rakić, and J. M. Elazar, "Modeling the optical constants of solids using acceptance-probabilitycontrolled simulated annealing with an adaptive move generation procedure," Phys. Rev. E 55, 4797-48903 (1997).

39. J. Märtensson and H. Arwin, "Applications of derivative lineshape fitting to ellipsometric spectra of thin films of metalsubstituted phthalocyanines," Thin Solid Films 205, 252-257 (1991).

40. A. B. Djurišić, T. Fritz, and K. Leo, "Modelling the optical constants of organic thin films: impact of the choice of objective function," J. Opt. A 2, 458-464 (2000).

41. B. Masenelli, S. Callard, A. Gagnaire, and J. Joseph, "Fabrication and characterization of organic semiconductor-based microcavities," Thin Solid Films 364, 264-268 (2000).

42. Y. L. Pan, Y. J. Wu, L. B. Chen, Y. Y. Zhao, Y. H. Shen, F. M. Li, S. Y. Shen, and D. H. Huang, "Structure and spectroscopic characterization of polycrystalline vanadyl phthalocyanine (VOPc) films fabricated by vacuum deposition," Appl. Phys. A 66, 569-573 (1998).

43. P. N. Day, Z. Wang, and R. Pachter, "Calculation of the structure and absorption spectra of phthalocyanines in the gasphase and in solution," Thermochem. Acta 455, 33-50 (1998).

44. J. Godlewski, J. Kalinowski, S. Stizza, I. Davoli, and R. Bernardini, "Asymmetries in the optical properties of vacuumdeposited organic films illuminated at the substrate and nonsubstrate surfaces," Thin Solid Films 146, 115-132 (1987). 